\title{
KEYWORD EXTRACTION BASED SUMMARIZATION OF CATEGORIZED KANNADA TEXT DOCUMENTS
}

\author{
Jayashree. $\mathrm{R}^{1}$, Srikanta Murthy. $\mathrm{K}^{2}$ and Sunny. $\mathrm{K}^{1}$ \\ ${ }^{1}$ Department of Computer Science, PES Institute Of Technology, Bangalore, India \\ jayashree@pes.edu, sunfinite@gmail.com \\ ${ }^{2}$ Department of Computer Science, PES School Of Engineering, Bangalore, India \\ srikantamurthy@pes .edu
}

\begin{abstract}
The internet has caused a humongous growth in the number of documents available online. Summaries of documents can help find the right information and are particularly effective when the document base is very large. Keywords are closely associated to a document as they reflect the document's content and act as indices for a given document. In this work, we present a method to produce extractive summaries of documents in the Kannada language, given number of sentences as limitation. The algorithm extracts key words from pre-categorized Kannada documents collected from online resources. We use two feature selection techniques for obtaining features from documents, then we combine scores obtained by GSS (Galavotti, Sebastiani, Simi) coefficients and IDF (Inverse Document Frequency) methods along with TF (Term Frequency) for extracting key words and later use these for summarization based on rank of the sentence. In the current implementation, a document from a given category is selected from our database and depending on the number of sentences given by the user, a summary is generated.
\end{abstract}

\section{KEYWORDS}

Summary, Keywords, GSS coefficient, Term Frequency (TF), IDF (Inverse Document Frequency) and Rank of sentence

\section{Introduction}

With the growth of the internet, a large amount of data is available online. There is a demanding need to make effective use of data available in native languages. Information Retrieval [IR] is therefore becoming an important need in the Indian context. India is a multilingual country; any new method developed in IR in this context needs to address multilingual documents. There are around 50 million Kannada speakers and more than 10000 articles in Kannada Wikipedia. This warrants us to develop tools that can be used to explore digital information presented in Kannada and other native languages. A very important task in Natural Language Processing is Text Summarization. Inderjeet Mani provides the following succinct definition for summarization is: take an information source, extract content from it, and present the most important content to the user in a condensed form and in a manner sensitive to the user's application needs[14].There are two main techniques for Text Document Summarization: extractive summary and abstractive 
International Journal on Soft Computing ( IJSC ) Vol.2, No.4, November 2011

summary. While extractive summary copies information that is very important to the summary, abstractive summary condenses the document more strongly than extractive summarization and require natural language generation techniques. Summarization is not a deterministic problem, different people would chose different sentences and even the same person may chose different sentences at different times, showing differences between summaries created by humans. Also, semantic equivalence is another problem, because two sentences can give the same meaning with different wordings. In this paper, we present an extractive summarization algorithm which provides generic summaries. The algorithm uses sentences as the compression basis. Keywords/phrases, which are a very important component of this work, are nothing but expressions; single words or phrases describing the most important aspects of a given document. The list of keywords/phrases aims to reflect the meaning of the document. Guided by the given keywords/phrases, we can provide a quick summary, which can help people easily understand what a document describes, saving a great amount of time and thus money. Consequently, automatic text document summarization is in high demand. Meanwhile, summarization is also fundamental to many other natural language processing and data mining applications such as information retrieval, text clustering and so on [11][2].

\section{Literature Survey}

Previous work on key phrase extraction by Letian Wang and Fang Li [3] has shown that key phrase extraction can be achieved using chunk based method. Keywords of document are used to select key phrases from candidates. Similarly, another approach by Mari-SannaPaukkeri et al[2] selects words and phrases that best describe the meaning of the documents by comparing ranks of frequencies in the documents to the corpus considered as reference corpus. The SZETERGAK system by Gabor Berend[1] is a frame work that treats the reproduction of reader assigned keywords as a supervised learning task. In this work, a restricted set of token sequences was used as classification instances. One more method of You Ouyang[4] extracted the most essential words and then expanded the identified core words as the target key phrases by word expansion approach. A novel approach to key phrase extraction proposed by them consists of two stages: identifying core words and expanding core words to key phrases. The work of automatically producing key phrases for each scientific paper by Su Nam Kim et al[5] has compiled a set of 284 scientific articles with key phrases carefully chosen by both their authors and readers, the task was to automatically produce key phrases for each paper. FumiyoFukumoto[6] present a method for detecting key sentences from the documents that discuss the same event. To eliminate redundancy they use spectral clustering and classified each sentence into groups each of which consists of semantically related sentences. The work of Michael .J . Paul et al[7] use an unsupervised probabilistic approach to model and extract multiple viewpoints in text. The authors also use Lex rank, a novel random walk formulating to score sentences and pairs of sentences from opposite view points based on both representativeness of the collections as well as their contrast with each other. The word position information proves to play a significant role in document summarization. The work of You Ouyang [8] et al illustrates the use of word position information, the idea comes from assigning different importance to multiple words in a single document .Cross Language document summary is another upcoming trend that is growing in Natural Language Processing area, wherein the input document is in one language, the summarizer produces summary in another language. There was a proposal by Xiaojun Wan et al [9] to consider the translation from English to Chinese. First the translation quality of each English sentence in the document set is predicted with the SVM regression method and then the quality score of each sentence is incorporated into the summarization process; finally English sentences with high translation scores are translated to form the Chinese summary. There have 
International Journal on Soft Computing ( IJSC ) Vol.2, No.4, November 2011

been techniques which use $A^{*}$ algorithm to find the best extractive summary up to given length, which is both optimal and efficient to run. Search is typically performed using greedy technique which selects each sentence in the decreasing order of model score until the desired length summary is reached [10]. There are two approaches to document summarization, supervised and unsupervised methods. In supervised approach, a model is trained to determine if a candidate phrase is a key phrase. In unsupervised method graph based methods are state-of-the art. These methods first build a word graph according to word co occurrences within the document and then use random walk techniques to measure the importance of a word [12].

3. Methodology:The methodology adopted by us can be described in four major steps:

\subsection{Crawling}

The first step is creating the Kannada dataset. Wget, a Unix utility tool was used to crawl the data available on http://kannada.webdunia.com. Data was pre-categorized on this web site.

\subsection{Indexing}

Python was the language of choice. The indexing part consisted of removing HTML mark up; English words need not be indexed for our work. Beautiful Soup is a python HTML/XML parser which makes it very easy to scrape a screen. It is very tolerant with bad markup. We use Beautiful Soup to build a string out of the text on the page by recursively traversing the parse tree returned by Beautiful Soup. All HTML and XML entities (\&\#3205; :๑, \&lt; : <) are then converted to their character equivalents. Normal indexing operations involve extracting words by splitting the document at non-alphanumeric characters, however this would not serve our purpose because dependent vowels ( ๖ळ, ๖ etc.) are treated as non-alphanumeric, so splitting at nonalphanumeric characters would not have worked for tokenization. Hence a separate module was written for removing punctuations.

\subsection{Keyword Extraction}

Documents in five categories were fetched: sports, religion, entertainment, literature, astrology. The next step is to calculate GSS coefficients and the Inverse Document Frequency (IDF) scores for every word (in a given category in the former case) in a given document belonging to these categories. Every word in a given document has a Term Frequency (TF), which gives the number of occurrence of a term in a given document, defined by:

$\mathrm{TF}=$ frequency of a term in a document / number of terms in a given document.

Similarly, the IDF of a document is given by the formula,

$\mathrm{IDF}=\log _{10}(\mathrm{~N} / \mathrm{n})$, where ' $\mathrm{N}$ ' is the total number of documents indexed across all categories. and ' $n$ ' is the number of documents containing a particular term.

Hence TF and IDF are category independent. The GSS coefficients which evaluate the importance of a particular term to a particular category are calculated. GSS(Galavotti-Sebastiani- 
Simi) co-efficient [13] is a feature selection technique used as the relevance measure in our case. Given a word w and category c it

is defined as:

$\mathrm{f}(\mathrm{w}, \mathrm{c})=\mathrm{p}(\mathrm{w}, \mathrm{c})^{*} \mathrm{p}\left(\mathrm{w}^{\prime}, \mathrm{c}^{\prime}\right)-\mathrm{p}\left(\mathrm{w}^{\prime}, \mathrm{c}\right) * \mathrm{p}\left(\mathrm{w}, \mathrm{c}^{\prime}\right)$

where, $\mathrm{p}(\mathrm{w}, \mathrm{c})$ is the probability that a document contains word ' $\mathrm{w}$ ' and belongs to category ' $\mathrm{c}$ '

$\mathrm{p}\left(\mathrm{w}^{\prime}, \mathrm{c}^{\prime}\right)$ is the probability that a document does not contain ' $\mathrm{w}$ ' and does not belong to ' $\mathrm{c}$ '

$\mathrm{p}(\mathrm{w}$ ', $\mathrm{c})$ is the probability that a document does not contain ' $\mathrm{w}$ ' and belongs to ' $\mathrm{c}$ '

$\mathrm{p}(\mathrm{w}, \mathrm{c}$ ' $)$ is the probability that a document contains ' $\mathrm{w}$ ' and does not belong to ' $\mathrm{c}$ '.

GSS coefficients give us words which are most relevant to the category to which the documents belong. IDF gives us words which are of importance to the given documents independently. Thus using these two parameters to determine relevant parts of the document helps in providing a wholesome summary.

\subsection{Summarization}

Given a document and a limit on the number of sentences, we have to provide a meaningful summary. We calculate the GSS coefficients and IDF of all the words in the given document. If the document is already present in our database, GSS coefficients and IDF values are already calculated offline. These values are then multiplied by the TF of the individual words to determine their overall importance in the document. We then extract top $n$ keywords from each of the lists (GSS coefficients and IDF). Then sentences are extracted from the given document by retrieving Kannada sentences ending with full stops. Due care is taken to see that full stops which do not mark the end of a sentence (๘ా . etc.) are not considered as split points. Each of these sentences is then evaluated for the number of keywords it contains from the GSS and IDF lists as follows:

Rank of sentence $=$ Number of keywords contained by the sentence from both the lists

Total number of sentences in the document

The top ' $m$ ' sentences are then returned as document summary, where ' $m$ ' is the user specified limit.

The same concept can be extended to paragraphs to provide a possibly more meaningful context than individual sentences particularly in the case of large documents.

\subsection{Tests}

The following lists were obtained by running our algorithm with a sports article on cricket as input with $n=20$ : 
GSS co-efficient list:

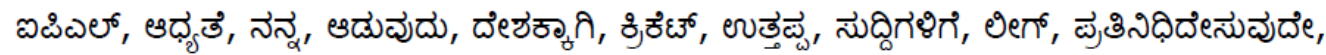

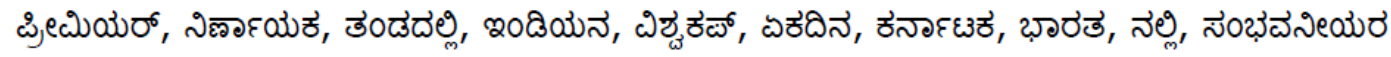

IDF list:

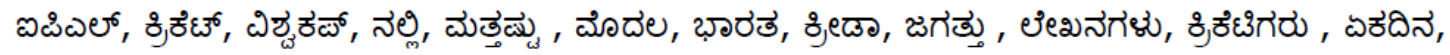

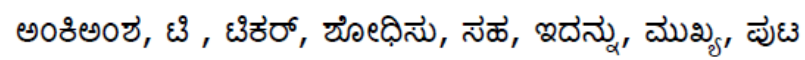

The following lists were obtained with a blog entry about another blog on films (category: literature) as input with $n=20$ :

GSS co-efficient list:

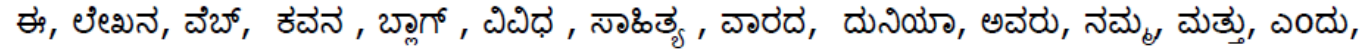

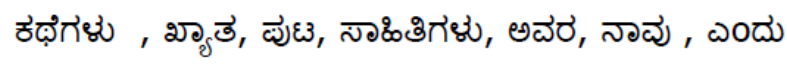

\section{IDF list:}

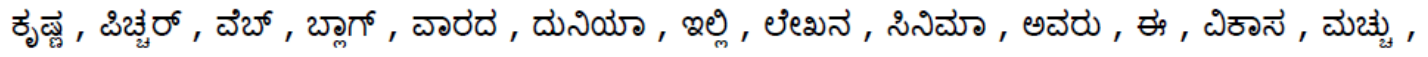

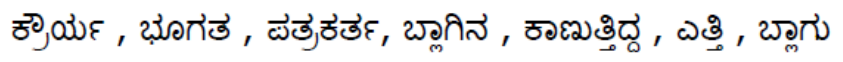

As evident, these lists contain stop words and hence, stop word (noise) removal is essential.

\subsection{Noise Removal}

Stop words which do not give meaning to the document considered noise and hence should not be evaluated as keywords. To remove stop words we have implemented an algorithm which takes a list of stop words prepared manually as input and finds structurally similar words and adds them to the stop word list.

Some of the words in our primary list of stop word which is created and maintained manually are:

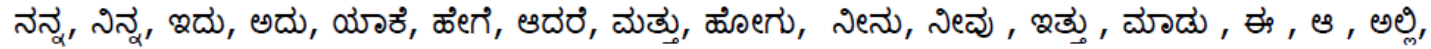

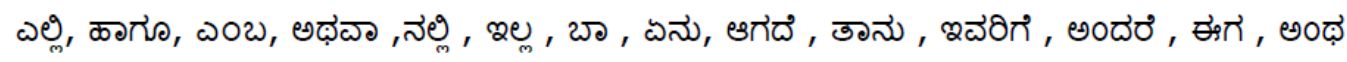

\subsubsection{Finding Structurally similar words for Primary list words}

Example: Consider the word ' యోळర్య '

When split into individual Unicode characters, 
it becomes : య (U+0CAF) + ๖ळ (U+0CBE) + శ (U+0C95) + ゃ(U+0CC6). The vowel sound at the end is not considered as an alphanumeric character. So our similarity module does the following in order :

1. Fuzzy search for words which contain the unmodified word at the beginning.

2. Strip all the non-alphanumeric characters at the end of the word and then fuzzy search for words which contain the modified word at the beginning.

A sample of stop words that were obtained by our algorithm :

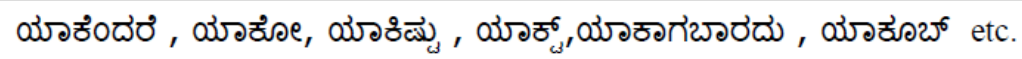

For the personal pronoun 'నెనెన', , some of the words obtained are:

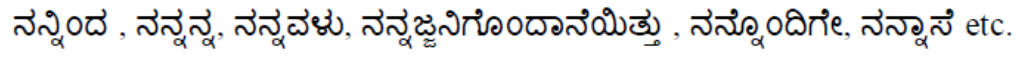

For the verb ' $బ ठ ి '$, the similar words were:

బరిసుదె, బరియ,, బరిది, బరిగాలల్లి,, బరిగణ్ణిగ, బరిగృలి, బరిగాలినెల్లి, బరిగాలల్లి, బరిగృయల్లి,

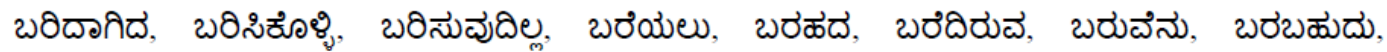
బరియుదుదిల్ల, బరిదిద్ద, బరియిరి, బరెలిల, బరుదరు, బరెడగగారిగిగి, బరుతిలర, బరియలారురు, బరిదుకోంండె, బరియబబల్లరు, బతికల్టల్ల etc.

As evident, though some words have semantic relationship to the primary stop word, a lot of words have no such relationship and further work needs to be done to find methods which will prevent such words from being penalized as stop words. Starting with a basic list of stop words, this program can be used to find structurally similar words and semantically unrelated words can be manually removed from the stop words list.

\subsection{Results}

Evaluation-I gives the result of a manual evaluation of the summarizer with three different human summaries across various categories. Three different people were asked to create reference summaries for random documents in each category. The same documents were then fed to the program and the limit was kept at $\mathrm{m}=10$. The number of sentences common between the two summaries gives the relevance score; the average of three scores is shown for each document.

Evaluation-II consisted of a single human reference summary for a data set consisting of 20 
International Journal on Soft Computing ( IJSC ) Vol.2, No.4, November 2011

documents across different categories. This human reference summary was compared to the summary generated by the machine.For Evaluation III and Evaluation IV, we considered 20 documents, across categories: Literature, Entertainment, Sports, and Religion. Two independent reviewers were asked to summarize the documents (not Abstractive), the results are shown in Table 3 and Table 4.

Evaluation V and Evaluation VI given in Table 5 and Table 6 shows the number of common sentences between human summary and Machine summary. Wherein machine summary is generated for the same set of documents across same categories considered for Evaluation III and Evaluation IV. The maximum limit on the number of sentences to be given by the machine is the number of sentences obtained by human summarizers for a given document.

Evaluation VII and Evaluation VIII consists of the average of the human summary and machine summary, means the number of sentences common between the human summary and machine summary is taken into consideration and average is taken.

Table 1. Evaluation-I Results.

\begin{tabular}{|l|l|l|l|l|}
\hline Category & Human 1 Score & Human 2 Score & Human 3 Score & Average \\
\hline Literature & 0.4 & 0.5 & 0.2 & 0.37 \\
\hline Entertainment & 0.5 & 0.2 & 0.6 & 0.43 \\
\hline Astrology & 0.8 & 0.7 & 0.7 & 0.73 \\
\hline Sports & 0.4 & 0.4 & 0.4 & 0.40 \\
\hline
\end{tabular}

Table 2.Evaluation-II Results.

\begin{tabular}{|l|l|}
\hline Category & Score \\
\hline Literature & 0.7 \\
\hline Entertainment & 0.8 \\
\hline Astrology & 0.8 \\
\hline Sports & 0.76 \\
\hline
\end{tabular}


International Journal on Soft Computing ( IJSC ) Vol.2, No.4, November 2011

Table 3.Evaluation-III

\begin{tabular}{|l|l|l|l|l|l|l|l|l|l|l|}
\hline & D1 & D2 & D3 & D4 & D5 & D6 & D7 & D8 & D9 & D10 \\
\hline Literature & $30 / 32$ & $34 / 37$ & $13 / 18$ & $32 / 37$ & $20 / 26$ & $13 / 17$ & $19 / 23$ & $20 / 22$ & $29 / 35$ & $12 / 17$ \\
\hline Entertainment & $11 / 16$ & $02 / 04$ & $14 / 18$ & $13 / 19$ & $10 / 16$ & $04 / 09$ & $19 / 25$ & $06 / 12$ & $09 / 15$ & $07 / 13$ \\
\hline Sports & $11 / 17$ & $07 / 11$ & $08 / 14$ & $14 / 16$ & $12 / 13$ & $10 / 15$ & $14 / 20$ & $07 / 13$ & $02 / 05$ & $07 / 15$ \\
\hline Religion & $13 / 15$ & $08 / 11$ & $03 / 03$ & $08 / 11$ & $13 / 17$ & $18 / 20$ & $06 / 08$ & $04 / 06$ & $09 / 10$ & $19 / 21$ \\
\hline
\end{tabular}

Table 4.Evaluation- IV

\begin{tabular}{|l|l|l|l|l|l|l|l|l|l|l|}
\hline & D1 & D2 & D3 & D4 & D5 & D6 & D7 & D8 & D9 & D10 \\
\hline Literature & $07 / 12$ & $12 / 14$ & $25 / 28$ & $01 / 01$ & $19 / 22$ & $31 / 35$ & $19 / 24$ & $19 / 22$ & $17 / 20$ & $11 / 14$ \\
\hline Entertainment & $06 / 11$ & $04 / 08$ & $05 / 08$ & $25 / 27$ & $01 / 03$ & $06 / 12$ & $06 / 12$ & $06 / 12$ & $09 / 14$ & $03 / 08$ \\
\hline Sports & $20 / 22$ & $11 / 17$ & $04 / 09$ & $10 / 16$ & $05 / 09$ & $10 / 22$ & $10 / 19$ & $13 / 28$ & $41 / 50$ & $42 / 52$ \\
\hline Religion & $11 / 13$ & $06 / 08$ & $10 / 13$ & $01 / 01$ & $10 / 12$ & $16 / 20$ & $12 / 15$ & $06 / 07$ & $01 / 01$ & $12 / 13$ \\
\hline
\end{tabular}

Table 5.Evaluation $-\mathrm{V}$

\begin{tabular}{|l|l|l|l|l|l|l|l|l|l|l|}
\hline & D1 & D2 & D3 & D4 & D5 & D6 & D7 & D8 & D9 & D10 \\
\hline Literature & $03 / 07$ & $06 / 12$ & $18 / 25$ & $01 / 01$ & $11 / 19$ & $27 / 31$ & $11 / 19$ & $11 / 19$ & $10 / 17$ & $08 / 11$ \\
\hline Entertainment & $03 / 06$ & $02 / 04$ & $02 / 05$ & $18 / 25$ & $01 / 01$ & $02 / 06$ & $02 / 06$ & $02 / 06$ & $03 / 09$ & $01 / 03$ \\
\hline Sports & $16 / 20$ & $08 / 11$ & $02 / 04$ & $04 / 10$ & $01 / 05$ & $06 / 10$ & $06 / 10$ & $09 / 13$ & $32 / 41$ & $34 / 42$ \\
\hline Religion & $08 / 11$ & $02 / 06$ & $04 / 10$ & $01 / 01$ & $04 / 10$ & $10 / 16$ & $08 / 12$ & $02 / 06$ & $01 / 01$ & $05 / 12$ \\
\hline
\end{tabular}


International Journal on Soft Computing ( IJSC ) Vol.2, No.4, November 2011

Table 6.Evaluation VI

\begin{tabular}{|l|l|l|l|l|l|l|l|l|l|l|}
\hline & D1 & D2 & D3 & D4 & D5 & D6 & D7 & D8 & D9 & D10 \\
\hline Literature & $03 / 07$ & $05 / 12$ & $18 / 25$ & $01 / 01$ & $10 / 19$ & $22 / 31$ & $10 / 19$ & $10 / 19$ & $11 / 17$ & $07 / 11$ \\
\hline Entertainment & $02 / 06$ & $01 / 04$ & $01 / 05$ & $17 / 25$ & $01 / 03$ & $02 / 06$ & $02 / 06$ & $02 / 06$ & $02 / 09$ & $01 / 03$ \\
\hline Sports & $15 / 20$ & $08 / 11$ & $01 / 04$ & $05 / 10$ & $01 / 05$ & $05 / 10$ & $05 / 10$ & $07 / 13$ & $32 / 41$ & $35 / 42$ \\
\hline Religion & $08 / 11$ & $01 / 06$ & $04 / 10$ & $01 / 01$ & $04 / 10$ & $10 / 16$ & $05 / 12$ & $01 / 06$ & $01 / 01$ & $05 / 12$ \\
\hline
\end{tabular}

Table 7. Evaluation VII

\begin{tabular}{|l|l|l|l|l|l|l|l|l|l|l|}
\hline & D1 & D2 & D3 & D4 & D5 & D6 & D7 & D8 & D9 & D10 \\
\hline Literature & 0.42 & 0.50 & 0.72 & 100 & 0.81 & 0.87 & 0.81 & 0.81 & 0.58 & 0.72 \\
\hline Entertainment & 0.50 & 0.50 & 0.4 & 0.72 & 100 & 0.34 & 0.34 & 0.34 & 0.34 & 0.34 \\
\hline Sports & 0.8 & 0.72 & 0.50 & 0.40 & 0.20 & 0.6 & 0.6 & 0.70 & 0.78 & 0.80 \\
\hline Religion & 0.72 & 0.34 & 0.4 & 100 & 0.4 & 0.62 & 0.67 & 0.34 & 100 & 0.42 \\
\hline
\end{tabular}

Table 8. Evaluation VIII

\begin{tabular}{|l|l|l|l|l|l|l|l|l|l|l|}
\hline & D1 & D2 & D3 & D4 & D5 & D6 & D7 & D8 & D9 & D10 \\
\hline Literature & 0.25 & 0.42 & 0.72 & 100 & 0.52 & 0.70 & 0.52 & 0.70 & 0.64 & 0.63 \\
\hline Entertainment & 0.34 & 0.25 & 0.2 & 0.68 & 0.34 & 0.34 & 0.34 & 0.34 & 0.23 & 0.34 \\
\hline Sports & 0.75 & 0.72 & 0.25 & 0.5 & 0.2 & 0.5 & 0.5 & 0.53 & 0.78 & 0.83 \\
\hline Religion & 0.72 & 0.16 & 0.4 & 100 & 0.4 & 0.62 & 0.41 & 0.16 & 100 & 0.41 \\
\hline
\end{tabular}


International Journal on Soft Computing ( IJSC ) Vol.2, No.4, November 2011

Below is a snap shot of the summarizer output and the actual document in that order.
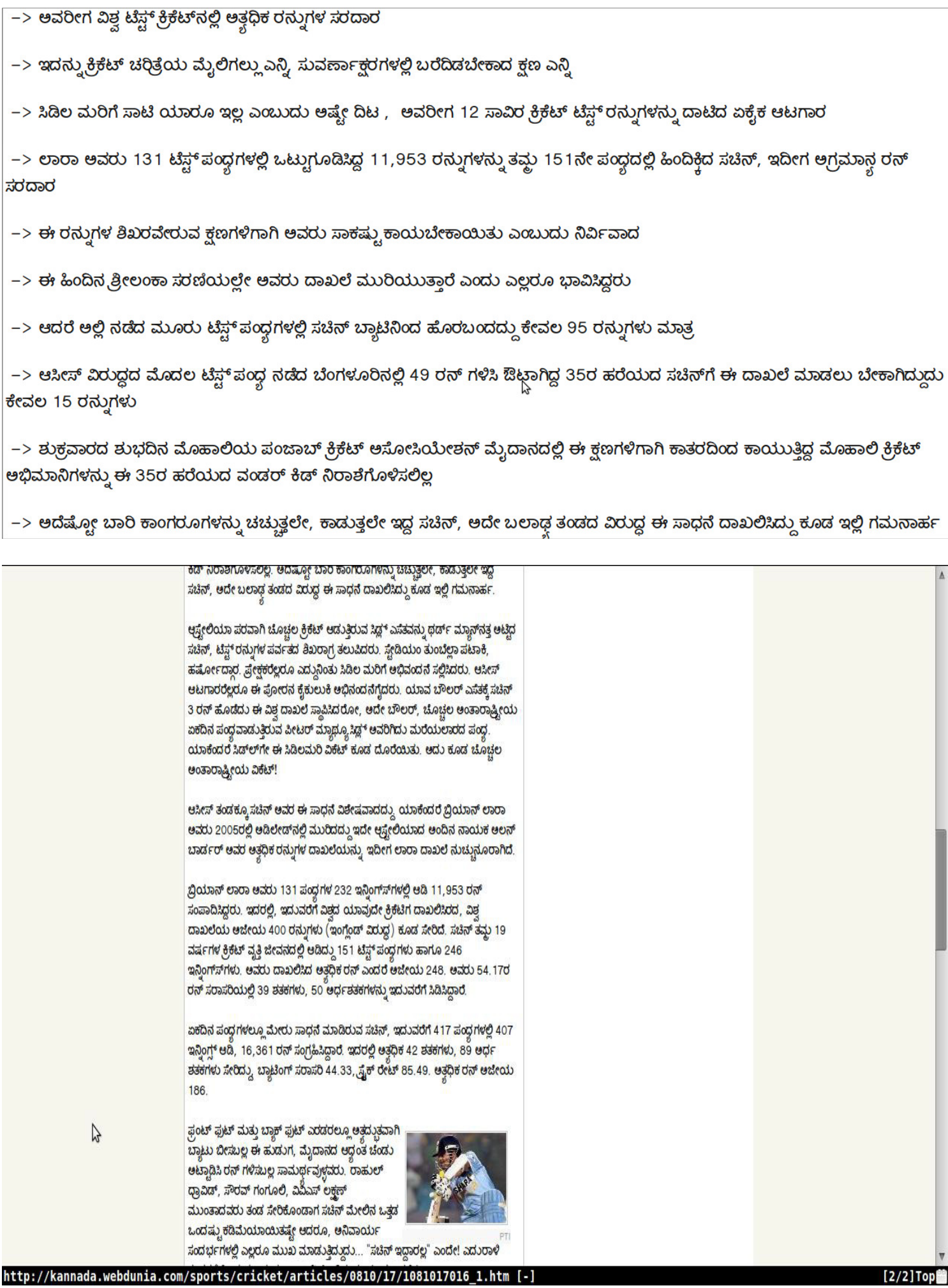
International Journal on Soft Computing ( IJSC ) Vol.2, No.4, November 2011

\subsection{Summarization of unclassified documents}

The main purpose of classification in our work is to make efficient use of feature selection techniques such as GSS co-efficient for summarizing documents. The same feature selection techniques can be used to train classifiers thereby improving the efficiency of classifiers.

When an unclassified document is given as input, the stop word removal method described above is applied. It is then submitted to the classifier which generates relevance scores depending on the number of GSS co-efficient keywords the particular document contains. Such a method will ensure that the classifier does not train itself on and is not misguided by frequently occurring but non-relevant terms to a category. Once a document is classified, the procedure outlined above can be followed.

If all the values returned by the classifier across all categories fall below the threshold, TF-IDF can be used as the fallback for summarization.

\section{Conclusion}

It is evident that evaluating summaries is a difficult task, as it is not deterministic. Different people may choose different sentences and also same people may choose different sentences at different times. Sentence recall measure is used as the evaluation factor. Although humans can also cut and paste relevant information of a text, most of the times they rephrase sentences when necessary, or they join different related information into one sentence. Hence paraphrasing and rephrasing are the issues in summarization. However in our test, sentences were selected as a whole both by the machine and human summarizers

Most Evaluation systems in Summarization require at least one reference summary, which is the most difficult and expensive task. Much effort is needed in order to have corpus of texts and their corresponding summaries.

The Classifier helps determine the relevant category. Hence any document given as input is able to produce the summary. There is no standard stop word list for Kannada, or methods to do that. Hence a given procedure in this work can be used as a stop word removal tool using structurally similar word removal technique. The summarizer can be used as a tool in various organizations such as Kannada Development Authority, Kannada SahityaParishath etc.

\section{References}

[1] Gabor Berend,Rich`ardFarkasSZTERGAK : Feature Engineering for Keyphrasextraction,Proceedings of the 5th International Workshop on Semantic Evaluation, ACL 2010, pp 186-189,Uppsala,Sweden, 15-16 July 2010.

[2] Mari-SannaPaukkeri and TimoHonkela,'Likey: Unsupervised Language-independent KeyphraseExtraction,Proceedings of the 5th International Workshop on Semantic Evaluation, ACL 2010, pp 162-165,Uppsala, Sweden, 15-16 July 2010.

[3] Letian Wang, Fang Li, SJTULTLAB: Chunk Based Method for Keyphrase Extraction, Proceedings of the 5th International Workshop on Semantic Evaluation, ACL 2010,pp 158- 161,Uppsala, Sweden, 15-16 July 2010. 
International Journal on Soft Computing ( IJSC ) Vol.2, No.4, November 2011

[4] You OuyangWenjie Li Renxian Zhang,'273. Task 5.Keyphrase Extraction Based on Core Word Identification and Word Expansion',Proceedings of the 5th International Workshop on Semantic Evaluation, ACL 2010, pp 142-145, Uppsala, Sweden, 15-16 July 2010.

[5] Su Nam Kim,ÄOlenaMedelyan, Min-Yen Kan\} and Timothy BaldwinÄ,'SemEval-2010 Task 5: Automatic Keyphrase Extraction from Scientific Articles',Proceedings of the 5th International Workshop on Semantic Evaluation, ACL 2010, pp 21-26,Uppsala, Sweden, 15-16 July 2010.

[6] FumiyoFukumotoAkina Sakai Yoshimi Suzuki,'Eliminating Redundancy by Spectral Relaxation for Multi-Document Summarization',Proceedings of the 2010 Workshop on Graph-based Methods for Natural Language Processing, ACL 2010, pp 98-102,Uppsala, Sweden, 16 July 2010.

[7] Michael J. Paul,ChengXiangZhai,RoxanaGirju,'Summarizing Contrastive Viewpoints in Opinionated Text',Proceedings of the 2010 Conference on Empirical Methods in Natural Language Processing, pp 66-76,MIT, Massachusetts, USA, 9-11 October 2010.

[8] You Ouyang, Wenjie Li Qin Lu Renxian Zhang, 'A Study on Position Information in Document Summarization', Coling 2010: Poster Volume, pp 919-927, Beijing, August 2010.

[9] Xiaojun Wan, Huiying Li and JianguoXiao,'Cross-Language Document Summarization Based on Machine Quality Prediction',Proceedings of the 48th Annual Meeting of the Association for Computational Linguistics, pp 917-926,Uppsala, Sweden, 11-16 July 2010.

[10] Ahmet Aker Trevor Cohn,'Multi-document summarization using $\mathrm{A} *$ search and discriminative training',Proceedings of the 2010 Conference on Empirical Methods in Natural Language Processing, pp 482-491,MIT, Massachusetts, USA, 9-11 October 2010.

[11] Hal Daum'e III*,DanielMarcu*,'Induction ofWord and Phrase Alignments for Automatic Document Summarization', Computational Linguistics, 31 (4), pp. 505-530, December, 2006.

[12] ZhiyuanLiu,Wenyi Huang, YabinZheng and MaosongSun,'AutomaticKeyphrase Extraction via Topic Decomposition',Proceedings of the 2010 Conference on Empirical Methods in Natural Language Processing, pp 366-376,MIT, Massachusetts, USA, 9-11 October 2010.

[13] L. Galavotti, F. Sebastiani, and M. Simi,'Experiments on the use of feature selection and negative evidence in automated text categorization' Proc. 4th European Conf. Research and AdvancedTechnology for Digital Libraries,SpringerVerlag,pp.59-68,2000.

[14] Automatic Summarization by Inderjeet Mani, John Benjamins Publishing Co. 


\section{Authors}

Jayashree.Ris an Asst.Professor in the Department of Computer Science and Engineering, at PES Institute of Technology, Bangalore. She has over 15 Years of teaching experience. She has published several papers in international conferences. Her research area of interest is Natural Language Processing and Pattern Recognition.

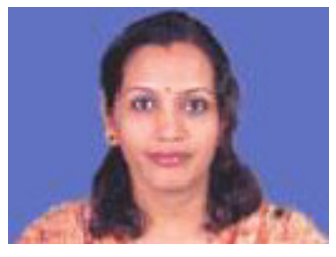

Dr.K.Srikanta Murthy is a Professor and Head, Department of Computer Science and Engineering, at PES School of Engineering, Bangalore. He has put in 24 years of service in teaching and 5 years in research. He has published 8 papers in reputed International Journals; 41 papers at various International Conferences. He is currently guiding $5 \mathrm{PhD}$ students . He is also a member of various Board of Studies andBoard of Examiners for different universities. His research areas include Image Processing ,Document Image analysis, Pattern

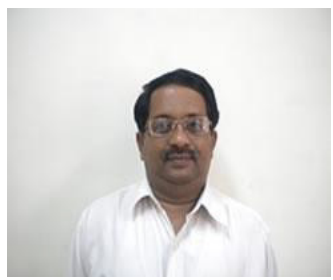
Recognition, Character Recognition ,Data Mining and Artificial Intelligence.

Sunny.Kis a final year Computer Science undergraduate student. 